\title{
Electropolymerisation of Phenylacetylene on Pt Electrodes with Propylene Carbonate and Acetonitryle as Solvents
}

\author{
J. García Cañadas, ${ }^{a}$ A. Lafuente, ${ }^{b}$ G. Rodríguez, \\ M.L. Marcos, ${ }^{a}$ J. González Velasco ${ }^{a, *}$ \\ Universidad Autónoma de Madrid. Facultad de Ciencias. 28049 Madrid. España \\ ${ }^{a}$ Departamento de Química. ${ }^{b}$ Departamento de Química Orgánica
}

Received 2 April 2004; accepted in revised form 29 June 2004

\begin{abstract}
The electropolymerization of phenylacetylene on a Pt electrode has been carried out in two different solvents, acetonitryle and propylene carbonate. The kinetic study carried out leads to values in the kinetic parameters which are significantly different in both solvents. The values deduced for $n \alpha, Z$ (reaction order with respect to the phenylacetylene concentration) and activation enthalpy and entropy, were $0.75,1,25.3$ $\mathrm{kJ} \mathrm{mol}^{-1}$ and $-87.0 \mathrm{~J} \mathrm{~mol}^{-1} \mathrm{~K}^{-1}$, respectively, in acetonitrile, and $0.25,0.5,33.6 \mathrm{~kJ} \mathrm{~mol}^{-1}$ and $-105 \mathrm{~J} \mathrm{~mol}^{-1} \mathrm{~K}^{-1}$, when working in propylene carbonate. The discrepancy in the values can be explained as a consequence of the change in the initiation step of the polymerisation induced by the adsorption of the acetonitryle molecules. The product obtained was isolated, purified and characterised to be formed by a polyenic chain with a linear trans structure. The average molar mass of the product was 3095 , corresponding to a polymer formed from around 30 units of monomer.
\end{abstract}

Keywords: mechanistic study, electropolymerization, polyphenylacetylene.

\section{Introducción}

Los polienos de cadena $\pi$ conjugada son nuevos materiales sintéticos que se caracterizan por poseer propiedades físicas tales como fotoconductividad, susceptibilidad óptica no lineal o susceptibilidad magnética, las cuales los hacen atractivos para aplicaciones prácticas [1,2]. 
Las propiedades de los polienos se pueden modular variando su masa molar, geometría, grado de conjugación, grupos de fin de cadena y cambiando la distribución de pesos moleculares. Para conseguir estos propósitos se ha recurrido a polimerizaciones mediadas a través de la presencia de compuestos de metales de transición, como Mo ó Rh, o de acetilenos substituidos [1].

Compuestos del mismo tipo han sido sintetizados electroquímicamente por reducción galvanostática [3] o potenciostática [4] de fenilacetileno (FA), como en el caso de la electropolimerización de FA a polifenilacetileno (PFA) [5]. Este compuesto se caracteriza por poseer propiedades de conductividad eléctrica, magnéticas, y de líquido cristalino [6].

En este estudio se presentan los resultados obtenidos en la electropolimerización, llevada a cabo por vía oxidativa, del FA a PFA en dos disolventes diferentes. Uno de ellos, acetonitrilo, está formado por moléculas que se adsorben sobre el electrodo de Pt utilizado y que, por lo tanto, impiden la adsorción del monómero, por lo que el iniciador de la electropolimerización se produce en disolución y continúa en el seno de la misma. El otro, carbonato de propileno, es menos propenso a adsorberse, lo que posibilita que la etapa de iniciación de la electropolimerización pueda tener lugar en la capa adsorbida, dando lugar a un cambio en los valores de los parámetros cinéticos, es decir, en el mecanismo de reacción con respecto al que tiene lugar en el otro disolvente. De este modo es posible obtener ecuaciones de velocidad teórica que coinciden con las experimentales. Se deduce también de la caracterización de los electropolímeros obtenidos, que la masa molar promedio corresponde a la unión de alrededor de 30 unidades de polímero y que están constituidos por cadenas poliénicas lineales con estructura trans.

\section{Experimental}

\section{Reactivos y técnicas generales}

El monómero utilizado fue fenilacetileno (FA) suministrado por Aldrich, que se sometió a destilación a baja presión. Carbonato de propileno (CP) anhidro (Fluka) se conservó sobre tamices moleculares (5 ̊). Acetonitrilo (AN) anhidro 
al 98\% fue utilizado directamente. $\mathrm{LiClO}_{4}$ se utilizó como electrolito soporte (Aldrich). $\mathrm{H}_{2} \mathrm{O}$ se sometió al procesado Milli-Q. Todos los disolventes se secaron y destilaron previamente a su utilización.

Los espectros RMN se registraron por medio de un aparato Bruker AMX-300. Los desplazamientos químicos se midieron con relación al ${ }^{13} \mathrm{C}$ y los protones residuales del disolvente usado $\left(\mathrm{CDCl}_{3}\right)$ para ${ }^{13} \mathrm{C}$ y ${ }^{1} \mathrm{H}$, respectivamente. Los espectros infrarrojos se registraron en un Bruker Vector 22 Spectrometer. Los espectros de masas se registraron en un espectrómetro VG AutoSpec. Espectros MALDI-TOF se registraron en un espectrómetro Hewlett Packard 8453.

El silica gel que se utilizó fue Scharlau 60, de 0.04 a $0.006 \mathrm{~mm}$ para columna rápida (malla 230-400) y la identificación previa se llevó a cabo por medio de capas de silica gel Macherey-Nagel G/UV 254 .

\section{Electrosíntesis y purificación del polímero}

La electrosíntesis del polímero se realizó en una célula electroquímica de tres electrodos. Como contraelectrodo se empleó una red de $\mathrm{Pt}$ de área considerablemente mayor que la del electrodo de trabajo, mientras que un hilo de plata actuó como cuasirreferencia. El electrodo de trabajo fue un hilo de Pt del 99.99\% de pureza, previamente activado por ciclados voltamétricos en disolución acuosa de $\mathrm{H}_{2} \mathrm{SO}_{4} 0.5 \mathrm{M}$.

La disolución preparada consistió en $6 \mathrm{~mL}$ de carbonato de propileno (PC), o la misma cantidad de acetonitrilo, $0.1 \mathrm{M}$ en $\mathrm{LiClO}_{4}$ y $1 \mathrm{M}$ en fenilacetileno destilado. Para la síntesis se aplicaron unos 30 ciclos de voltametría cíclica a cuatro disoluciones de partida idénticas. La muestra final de la que se estudió su caracterización fue la suma de las cuatro disoluciones. Cada ciclo consistía en barridos de potencial a $\mathrm{v}=10 \mathrm{mV} / \mathrm{s}$ desde $0.0 \mathrm{~V}$ como potencial inicial hasta 2.6 V como límite anódico y llegando a $-1.3 \mathrm{~V}$ como límite catódico, acabando el ciclo en $0.0 \mathrm{~V}$.

En el barrido anódico es donde se produce la polimerización, observándose la caída de un sólido marrón desde el electrodo de trabajo que se disuelve 
rápidamente. Debido a ello la disolución inicialmente incolora adquiere al final una coloración marrón.

El barrido catódico hasta $-1.3 \mathrm{~V}$ se realiza para despasivar el electrodo, pues, de lo contrario, si no se llega a alcanzar potenciales tan negativos no se produce polímero en el siguiente ciclo anódico.

La muestra obtenida a partir de $\mathrm{PC}$ se lava con hexano y $\mathrm{H}_{2} \mathrm{O}$. El hexano es inmiscible con el PC y el $\mathrm{H}_{2} \mathrm{O}$, pero disuelve el fenilacetileno que haya podido quedar sin reaccionar y las fracciones más ligeras del polímero.

Para eliminar el PC, (proceso muy costoso ya que su punto de ebullición es muy alto $\left(238^{\circ} \mathrm{C}\right)$ y es soluble en la mayoría de los disolventes) se lava la muestra con $\mathrm{H}_{2} \mathrm{O}$. El $\mathrm{H}_{2} \mathrm{O}$ disuelve el $\mathrm{LiClO}_{4}$ usado como sal de fondo y es parcialmente soluble en PC. Al añadir $\mathrm{H}_{2} \mathrm{O}$ a la disolución se forma una emulsión por la precipitación del polímero, que es insoluble en $\mathrm{H}_{2} \mathrm{O}$. Para deshacer la emulsión se añade $\mathrm{CH}_{2} \mathrm{Cl}_{2}$, que disuelve el polímero y también el PC, pero es inmiscible con el $\mathrm{H}_{2} \mathrm{O}$. Cuando la cantidad de $\mathrm{PC}$ disminuyó tras varios lavados con $\mathrm{H}_{2} \mathrm{O}$ en presencia de $\mathrm{CH}_{2} \mathrm{Cl}_{2}$, se realizó un lavado con hexano que se siguió por placa cromatográfica para asegurar la eliminación de todo el fenilacetileno que quedaba sin reaccionar.

El lavado con $\mathrm{H}_{2} \mathrm{O}$ para eliminar el $\mathrm{PC}$ se realizó hasta que se dejaron de observar las señales del PC por ${ }^{1} \mathrm{HRMN}$. Finalmente la disolución resultante se rota para eliminar el $\mathrm{CH}_{2} \mathrm{Cl}_{2}$ y se obtiene un sólido de color marrón que se caracterizó por ${ }^{1} \mathrm{HMRN},{ }^{13} \mathrm{CRMN},{ }^{1} \mathrm{H}_{-}{ }^{13} \mathrm{C}$ (HMQC) e IR.

En el caso de la electropolimerización desde acetileno la muestra obtenida se deja evaporar a temperatura ambiente. El sólido que queda se disuelve en acetona y se lleva a una columna cromatográfica de gel de sílice. Se utiliza tolueno como eluyente, con el que se separan dos componentes de la muestra, que se analizan por ${ }^{1} \mathrm{HRMN}$, viendo que en el primero se arrastra el fenilacetileno que no polimeriza junto a fracciones oligoméricas y señales alifáticas que también aparecen en el segundo componente. Un tercer componente se separa con etanol. Después de evaporar el disolvente se disuelve todo en cloroformo, excepto una pequeña fracción insoluble que se disuelve en acetona. Se observa por ${ }^{1} \mathrm{HRMN}$ 
que la parte soluble en cloroformo corresponde con la fracción polimérica mayoritaria.

Para purificar esta fracción se lava con hexano varias veces, obteniendo un aceite marrón viscoso insoluble en hexano, que se lava de nuevo con cloroformo, quedando finalmente un sólido marrón que se seca a vacío. Èste sólido se caracteriza por ${ }^{1} \mathrm{HMRN},{ }^{13} \mathrm{CRMN}$ y HMQC.

\section{Medidas electroquímicas}

Para realizar las medidas electroquímicas se utilizó un sistema PAR Mo. 273 controlado por medio de un ordenador. Todos los experimentos se llevaron a cabo en una célula de tres electrodos bajo atmósfera de $\mathrm{N}_{2}$. Tanto en CP como en AN se prepararon disoluciones $0.1 \mathrm{M}$ de $\mathrm{LiClO}_{4}$ como electrolito soporte. Como electrodo de trabajo (ET) y contraelectrodo (CE) se utilizaron hilos de Pt y el electrodo de referencia fue un hilo de $\mathrm{Ag}$ en $\mathrm{AN}$ y un electrodo de $\mathrm{Ag} \mid \mathrm{AgCl}$ en CP.

\section{Resultados y discusión}

\section{Caracterización de los polímeros obtenidos en CP y AN}

El PFA puede presentarse adoptando cuatro estructuras diferentes según sean la configuración de los dobles enlaces $\mathrm{C}=\mathrm{C}$ y la conformación de los enlaces sencillos C-C en la cadena principal del polímero. Dos de ellas son cis (ciscisoidal y cis-transoidal) y dos son trans (trans-transoidal y trans-cisoidal) [1]. Los espectros ${ }^{1} \mathrm{H}$ RMN obtenidos con los polímeros resultantes de la electropolimerización en $\mathrm{CP}$ y en AN presentaban en ambos casos una estructura similar con una señal amplia centrada entre 7.4 y 7.5 ppm en CP (Fig. 1a) y 7.2 y 7.4 ppm en AN (Fig. 1b), lo que indica la presencia de protones unidos a dobles enlaces [7]. La presencia de una cadena extendida y, más probablemente, la posición 1,3 de los grupos fenilos a lo largo del polímero puede ser la razón que explique el ensanchamiento de la señal así como el desplazamiento químico debido a la presencia de protones olefínicos hacia valores mayores [4]. Dado que en las estructuras cis-transoidal los protones unidos a dobles enlaces producen 
una señal en el intervalo entre 5.6 y 5.7 ppm [1] y dicha señal no aparece en el espectro, se excluyó que el polímero tuviera una estructura cis. Tal exclusión viene confirmada por la ausencia en el espectro infrarrojo de la banda característica $-\mathrm{C}-\mathrm{H}$ de la estructura cis a $870 \mathrm{~cm}^{-1}$.

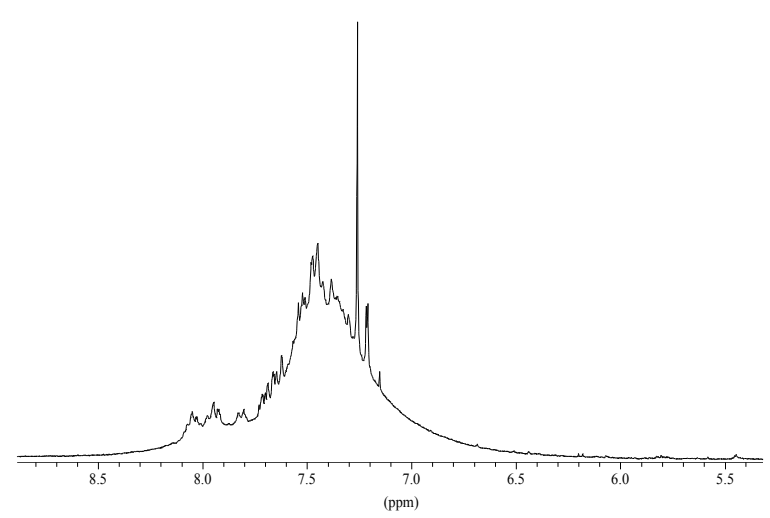

a)

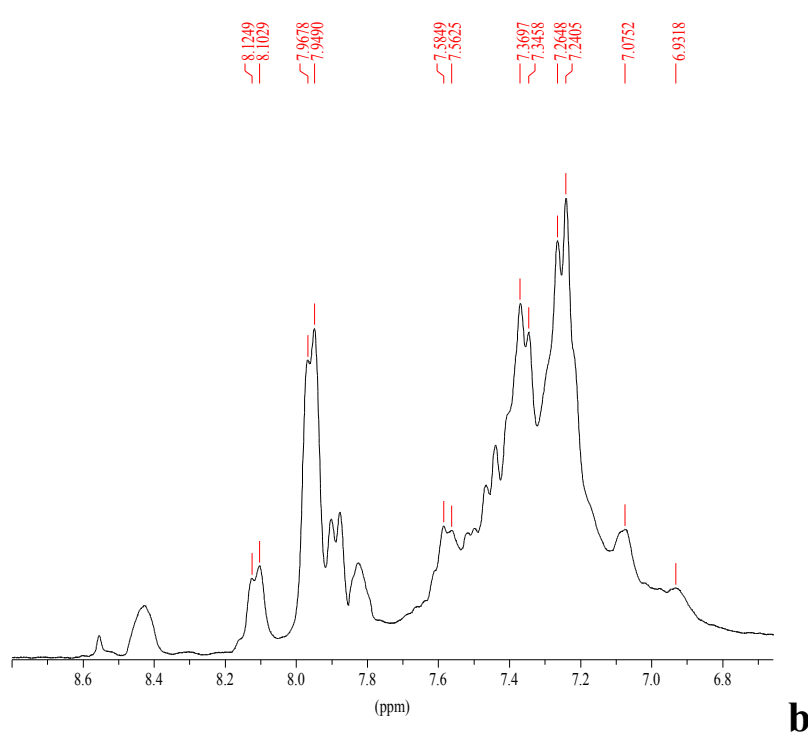

b)

Figura 1. a) Espectro ${ }^{1} \mathrm{H}$ RMN en $\mathrm{CDCl}_{3}$ de polifenilacetileno obtenido por electropolimerización en $\mathrm{CP}$; b) el mismo espectro para polifenilacetileno obtenido en acetonitrilo.

Los espectros IR de los productos de la electropolimerización en $\mathrm{CP}$ y $\mathrm{AN}$ aparecen en las Fig. 2a y 2b, respectivamente. 


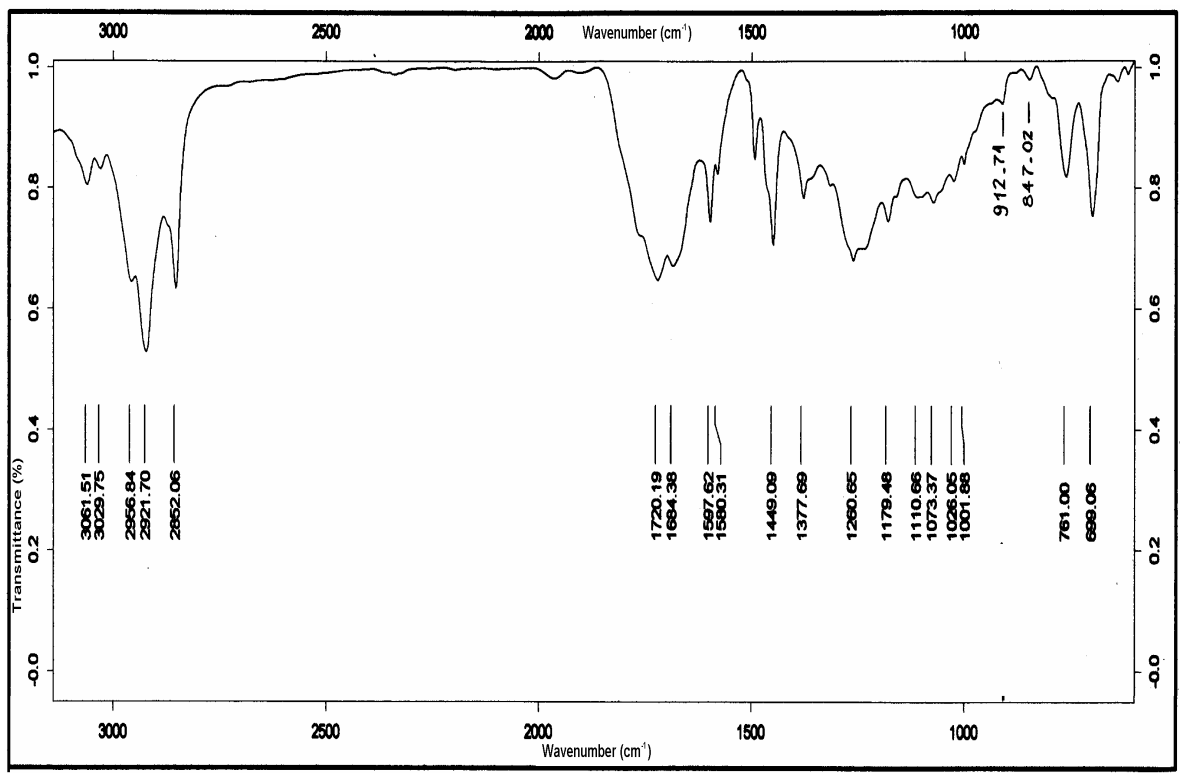

a)

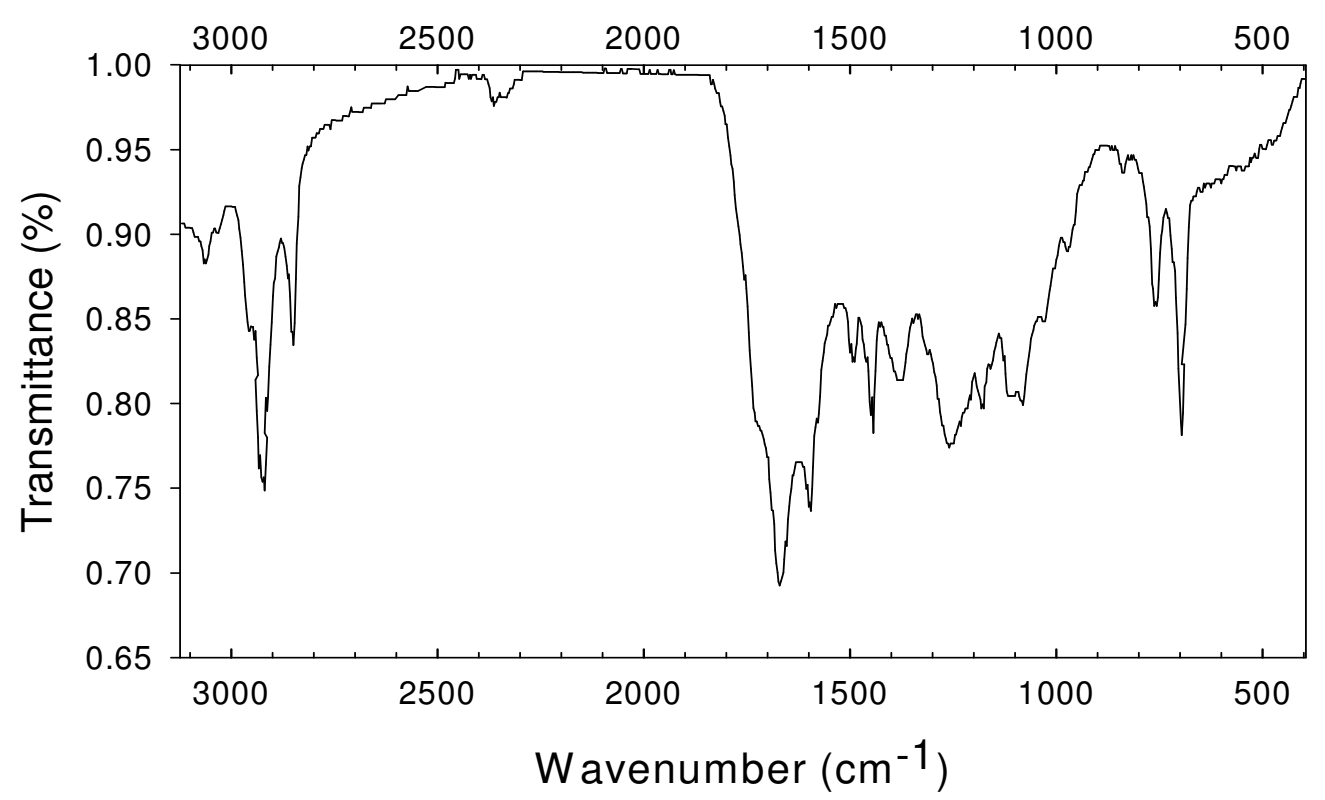

b)

Figura 2. a) Espectro IR de polifenilacetileno obtenido por electropolimerización en CP; b) el mismo espectro para polifenilacetileno obtenido en acetonitrilo.

A 1598 y $1580 \mathrm{~cm}^{-1}$ en CP y 1598 y $1669 \mathrm{~cm}^{-1}$ en AN aparecen las vibraciones de estiramiento características de los dobles enlaces conjugados y las debidas a la presencia de benceno monosubstituido a 761 y $699 \mathrm{~cm}^{-1}$ en CP y 762 y $700 \mathrm{~cm}^{-1}$ en AN. Esto permitió deducir que el producto de la polimerización en ambos 
medios presentaba una estructura de polieno lineal [8]. En CP aparece una banda de absorción a $913 \mathrm{~cm}^{-1}$ que se consideró característica de una estructura trans [9], mientras que en el espectro IR registrado a partir del polímero obtenido en AN aparecen tres bandas de absorción a 910,870 y $840 \mathrm{~cm}^{-1}$ que se atribuyen a insaturaciones en el producto.

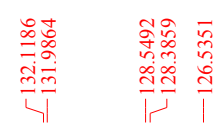

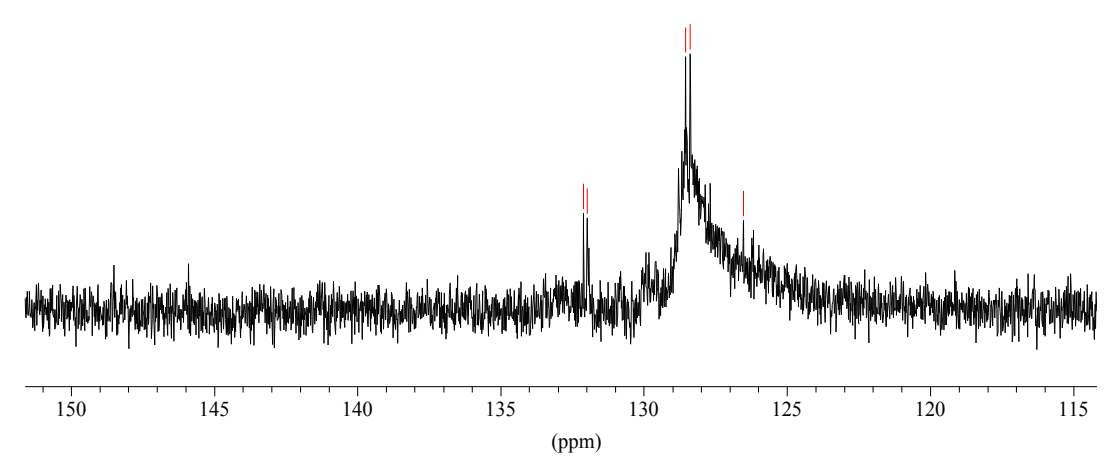

a)

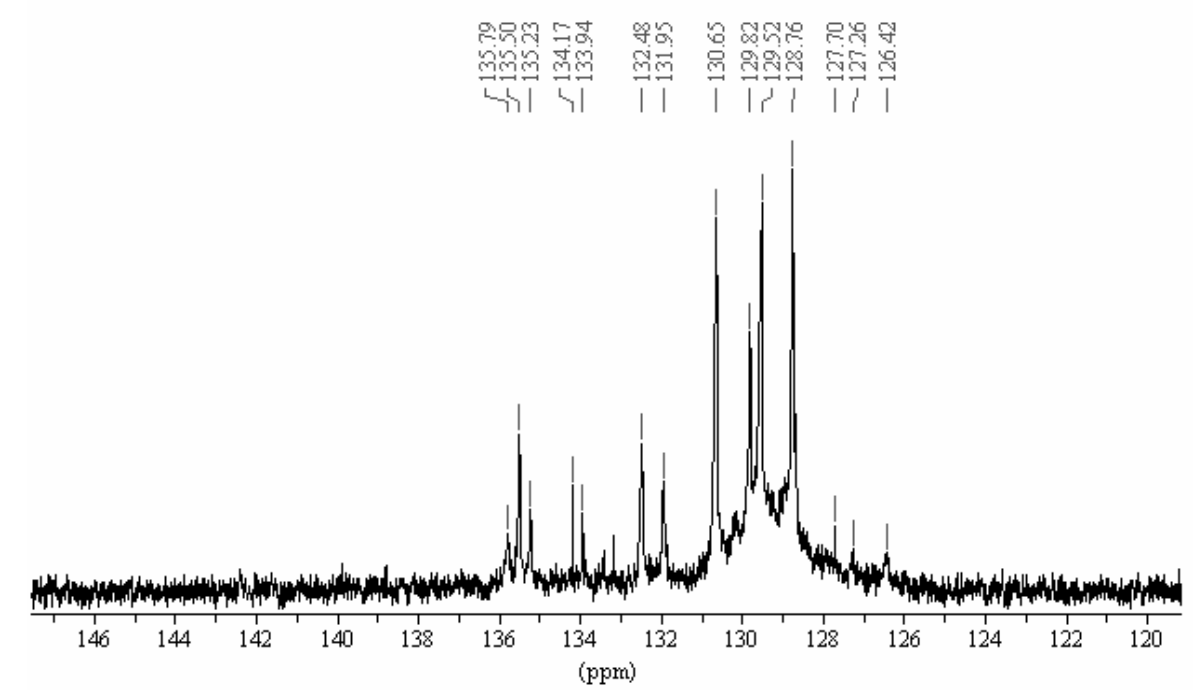

b)

Figura 3. a) Espectro ${ }^{13} \mathrm{C}$ RMN de polifenilacetileno obtenido por electropolimerización en $\mathrm{CP}$; b) el mismo espectro para polifenilacetileno obtenido en acetonitrilo. 
Las Fig. 3a y 3b muestran los espectros ${ }^{13} \mathrm{C}$ RMN en $\mathrm{CDCl}_{3}$ de los polímeros obtenidos en $\mathrm{CP}$ y AN, respectivamente. En ambos casos no pueden observarse las absorciones debidas a los carbonos cuaternarios, que aparecen a 142.8 y 139.2 ppm en compuestos olefínicos y aromáticos [10]. La estructura cis-cisoidal

presenta absorciones debidas a la presencia de carbonos $\mathrm{sp}^{3}$ que aparecen por formación de unidades de ciclohexadieno a 62.2 (para el carbono unido al hidrógeno) y 54.4 ppm [10] (para el carbono cuaternario) que no aparecen en ninguno de los dos espectros, lo que permite descartar la formación de un polímero con estructura cis-cisoidal. En CP aparecen dos singletes entre 127.8 y 126.4 ppm que pueden atribuirse a la estructura trans [10].

Por lo tanto, todos los datos espectroscópicos conducen a confirmar que el polímero sintetizado, tanto en $\mathrm{CP}$ como en $\mathrm{AN}$, tiene una estructura trans.

\section{Determinación de la masa molar media del polímero}

Con el fin de determinar la distribución de fracciones de polímero se utilizó la cromatografía de permeación de gel (HPLC-GPC). Se usaron columnas de $25 \mathrm{~cm}$ con tamaños de poro y partículas de $100 \AA$ y $5 \mu \mathrm{m}$, respectivamente. Como fase móvil se utilizó THF, aplicando un caudal de $1 \mathrm{~mL} / \mathrm{min}$, y el detector ultravioleta-visible medía en $\lambda=280 \mathrm{~nm}$. Las columnas se calibraron con muestras de poliestireno con números de masa media entre 3250 y 1320 [11].

Las muestras de polifenilacetileno dan lugar a una curva bimodal a $\mathrm{t}=6.18 \mathrm{~min}$, que corresponde en ambos disolventes a una masa molar promedio de 3095, es decir, el polímero formado corresponde a la unión de alrededor de 30 unidades de monómero. Como la formación de este polímero se produce, en $\mathrm{CP}$ y $\mathrm{AN}$, de acuerdo con los parámetros cinéticos y el mecanismo propuesto en los siguientes apartados, tras la formación de dos radicales feniletinilo, uno implicado en la reacción de poliadición radicálica que conduce a la formación de cada unidad de polímero y otro que da lugar a la reacción de terminación, se deduce que, por mol de electrones consumido se forman $1547 \mathrm{~g}$ de polímero, es decir, la mitad de la masa molecular media. La determinación de esta cantidad por culometría, no 
pudo llevarse a cabo porque el electrodo se pasiva a potenciales suficientemente positivos para producir polimerización anódica. Ello conduce a que para recuperar su capacidad electrocatalítica haya que someter al electrodo a pulsos de potencial negativo, como se describe en el apartado Electrosíntesis y purificación del polímero. Por otra parte, la ausencia de la banda de absorción característica del fenilacetileno en el espectro ultravioleta-visible del polímero llevó a la conclusión de que el monómero se convierte en polímero de forma cuantitativa.

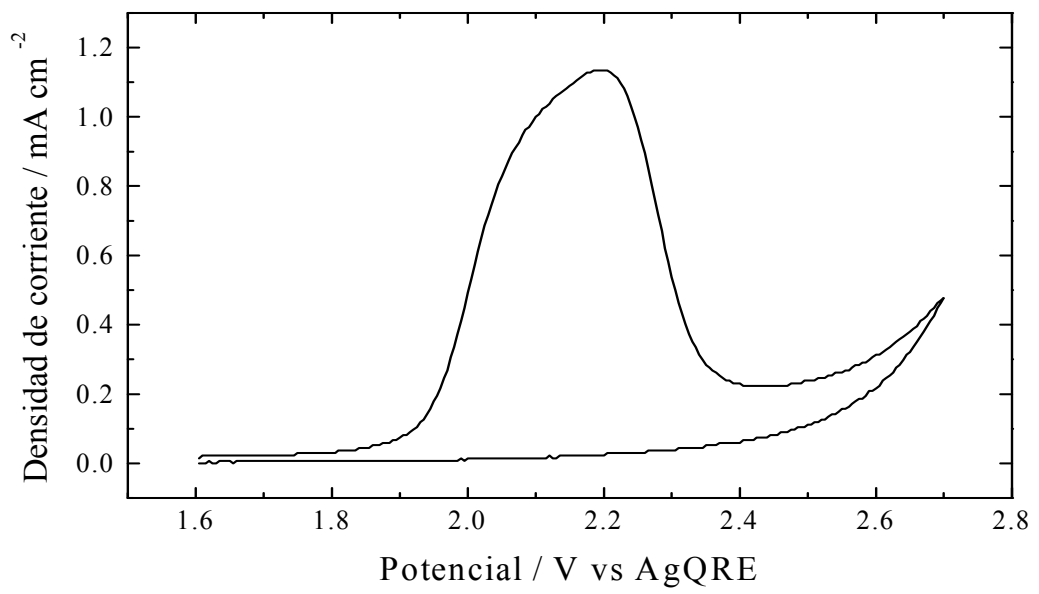

a)

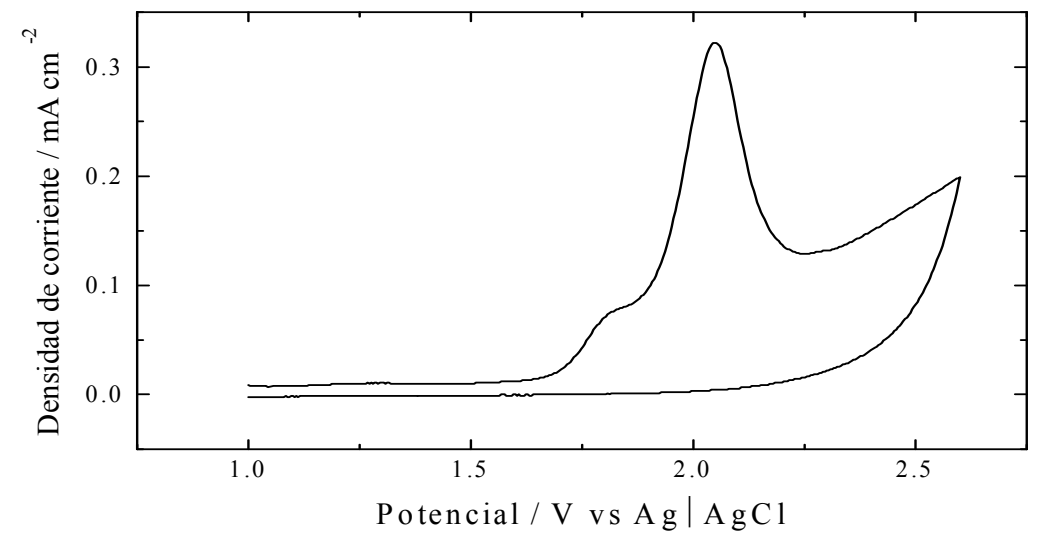

b)

Figura 4. a) Voltamograma registrado a $\mathrm{v}=10 \mathrm{mV} / \mathrm{s}$ en la electropolimerización de fenilacetileno en carbonato de propileno. $[\mathrm{FA}]=0.1 \mathrm{M}, \mathrm{T}=25^{\circ} \mathrm{C}$. Electrolito soporte $\mathrm{LiClO}_{4}$ 0.1 M. b) Lo mismo para la electropolimerización en acetonitrilo. 


\section{Estudio del mecanismo de electropolimerización}

El electrodo de trabajo (hilo de Pt) fue sometido, antes de realizar cualquier experimento electroquímico, a un ciclado repetitivo de potencial entre $0.05 \mathrm{y}$ $1.50 \mathrm{~V}_{\text {SHE }}(\mathrm{SHE}=$ Standard Hydrogen Electrode) en una disolución acuosa de $\mathrm{H}_{2} \mathrm{SO}_{4}$ 0.5 M. La finalidad de esta operación era la de activar al electrodo y proceder a la determinación del área real del mismo por integración del área del voltamograma base que corresponde a la formación de la capa de hidrógeno adsorbido.

Las Fig. 4a y $4 \mathrm{~b}$ muestran los voltamogramas, registrados bajo condiciones cuasiestacionarias a una velocidad de barrido $\mathrm{v}=10 \mathrm{mV} / \mathrm{s}$, para la electropolimerización del fenilacetileno en $\mathrm{CP}$ y $\mathrm{AN}$, respectivamente (concentración de monómero en disolución $=0.1 \mathrm{M}, \mathrm{T}=25^{\circ} \mathrm{C}$ ). Se dedujo que se trabajaba en condiciones cuasiestacionarias porque no se observó cambios en la pendiente de las curvas de densidad de corriente, $j$, frente al potencial al cambiar las velocidades de barrido por debajo de $50 \mathrm{mV} / \mathrm{s}$. Las pendientes de Tafel medidas en ambos medios fueron:

$$
\begin{aligned}
& \mathrm{b}=\left(\frac{\partial \mathrm{E}}{\partial \log \mathrm{j}}\right)_{\mathrm{T},[\mathrm{FA}]}=\frac{2.303 \mathrm{RT}}{\mathrm{n} \alpha \mathrm{F}} \\
& =236 \mathrm{mV} / \text { década de corriente en } \mathrm{CP}, \mathrm{y}: \\
& =79 \mathrm{mV} / \text { década de corriente en } \mathrm{AN}
\end{aligned}
$$

valores que permiten deducir, a partir de la Eq. 1 que el producto n $\alpha$ es 0.25 en CP y 0.75 en AN.

Las pendientes de Tafel no mostraban variaciones significativas al cambiar la concentración del monómero en ambos disolventes, lo que permitió hacer una determinación del orden de reacción con respecto a dicha concentración, $\left.\mathrm{Z}_{[\mathrm{FA}}\right]$. Para ello se registraron los voltamogramas a $\mathrm{v}=10 \mathrm{mV} / \mathrm{s}$ en disoluciones con concentraciones de monómero variables desde 0.05 a $0.6 \mathrm{M}$ en $\mathrm{CP}$ y entre 0.03 y $0.5 \mathrm{M}$ en AN, manteniendo la temperatura constante a $25^{\circ} \mathrm{C}$. A partir de las pendientes de las representaciones gráficas de $\log j$ frente a $\log [\mathrm{FA}]$, se 
obtuvieron los valores siguientes para los órdenes de reacción con respecto a la concentación de FA:

$$
\begin{aligned}
& \mathrm{Z}_{[\mathrm{FA}]}=\left(\frac{\partial \log \mathrm{j}}{\partial \log [\mathrm{FA}]}\right)_{\mathrm{T}, \mathrm{E}}= \\
= & 0.5 \mathrm{en} \mathrm{CP}, \mathrm{y}: \\
= & 1 \text { en } \mathrm{AN}
\end{aligned}
$$

Con los valores de n $\alpha$ y de $Z_{[F A]}$ se pueden escribir las ecuaciones de velocidad experimentales, que resultan ser, expresadas en unidades electroquímicas:

$$
j=\mathrm{nF} \mathrm{k}_{\mathrm{elp}}[\mathrm{FA}]^{0.5} \exp \left(\frac{0.25 \mathrm{FE}}{\mathrm{RT}}\right)
$$

para la electropolimerización en $\mathrm{CP}$, donde $\mathrm{n}$ es el número total de electrones transferidos en el mecanismo completo de formación del polímero, $\mathrm{F}$ representa la constante de Faraday, y $k_{\text {elp }}$ la constante de velocidad de la electropolimerización en CP.

Análogamente, la ecuación de velocidad experimental para la electropolimerización en AN es:

$$
j=\mathrm{nFk}_{\mathrm{elp}}^{\prime}[\mathrm{FA}] \exp \left(\frac{0.75 \mathrm{FE}}{\mathrm{RT}}\right)
$$

donde $\mathrm{k}_{\mathrm{elp}}^{\prime}$ representa la constante de velocidad de electropolimerización en AN.

Con el fin de poder determinar los valores de la entalpía $\left(\Delta \mathrm{H}^{ \pm}\right)$y de la entropía $\left(\Delta \mathrm{S}^{ \pm}\right)$de activación para la electropolimerización de fenilacetileno en CP y en AN, las ecuaciones de velocidad experimentales se expresaron substituyendo en las Eqs. 3 y 4 la siguiente ecuación para el valor de $\mathrm{k}_{\mathrm{elp}}$ que coincide con el valor de una constante de velocidad para una transferencia monoelectrónica, la cual puede ser expresada del siguiente modo $[12,13]$ : 


$$
\mathrm{k}_{\mathrm{elp}}=\kappa\left(\frac{\mathrm{k}_{\mathrm{B}} \mathrm{T}}{2 \pi \mathrm{m}}\right)^{1 / 2} \exp \left(\frac{\Delta \mathrm{S}_{\mathrm{elp}}^{ \pm}}{\mathrm{R}}\right) \exp \left(-\frac{\Delta \mathrm{H}_{\mathrm{elp}}^{ \pm}}{\mathrm{RT}}\right)
$$

donde $\kappa$ representa el coeficiente de transmisión, el cual, para reacciones de transferencia adiabáticas, como se supone que debe comportarse la transferencia de carga monoelectrónica que actúa como iniciadora de los mecanismos de electropolimerización, es igual a la unidad, $\mathrm{k}_{\mathrm{B}}$ es la constante de Boltzmann, m la masa molecular de la especie electroactiva, $\mathrm{R}$ la constante de los gases, y $\Delta \mathrm{H}_{\mathrm{elp}}{ }^{*}$ e $\Delta \mathrm{S}_{\mathrm{elp}}{ }^{*}$, los valores de la entalpía y entropía de electropolimerización, respectivamente.

Substituyendo la Eq. (5) en la Eq. (4) y reorganizando se puede llegar a la siguiente ecuación formada por dos miembros adimensionales:

$$
\ln \left(\frac{\mathrm{j}}{\mathrm{T}^{1 / 2}} \frac{\left(2 \pi \mathrm{M}_{\mathrm{FA}}\right)^{1 / 2}}{\mathrm{knF}[\mathrm{FA}]_{[\mathrm{FA}}^{\mathrm{Z}}\left(\mathrm{k}_{\mathrm{B}}\right)^{1 / 2}}\right)=\left[\frac{\Delta \mathrm{S}_{\mathrm{elp}}^{ \pm}}{\mathrm{R}}\right]-\frac{1}{\mathrm{~T}}\left[\frac{\Delta \mathrm{H}_{\mathrm{elp}}^{ \pm}-\mathrm{n} \alpha \mathrm{FE}}{\mathrm{R}}\right]
$$

y que, considerando los parámetros cinéticos determinados en cada uno de los disolventes permite escribir las ecuaciones siguientes:

$$
\ln \left(\frac{\mathrm{j}}{\mathrm{T}^{1 / 2}} \frac{2.9 \times 10^{-6}}{\left(\mathrm{~A} \mathrm{~cm}^{-2} \mathrm{~K}^{-1 / 2}\right)}\right)=\left[\frac{\Delta \mathrm{S}_{\mathrm{elp}}^{ \pm}}{\mathrm{R}}\right]-\frac{1}{\mathrm{~T}}\left[\frac{\Delta \mathrm{H}_{\mathrm{elp}}^{ \pm}-0.25 \mathrm{FE}}{\mathrm{R}}\right]
$$

que permite determinar $\Delta \mathrm{S}_{\text {elp }}{ }^{ \pm}$e $\Delta \mathrm{H}^{ \pm}$elp en $\mathrm{CP}, \mathrm{y}$ :

$$
\ln \left(\frac{\mathrm{j}}{\mathrm{T}^{1 / 2}} \frac{1.45 \times 10^{-4}}{\left(\mathrm{~A} \mathrm{~cm}^{-2} \mathrm{~K}^{-1 / 2}\right)}\right)=\left[\frac{\Delta \mathrm{S}_{\mathrm{elp}}^{ \pm}}{\mathrm{R}}\right]-\frac{1}{\mathrm{~T}}\left[\frac{\Delta \mathrm{H}_{\mathrm{elp}}^{ \pm}-0.75 \mathrm{FE}}{\mathrm{R}}\right]
$$

de la que se obtienen las mismas magnitudes en AN. 


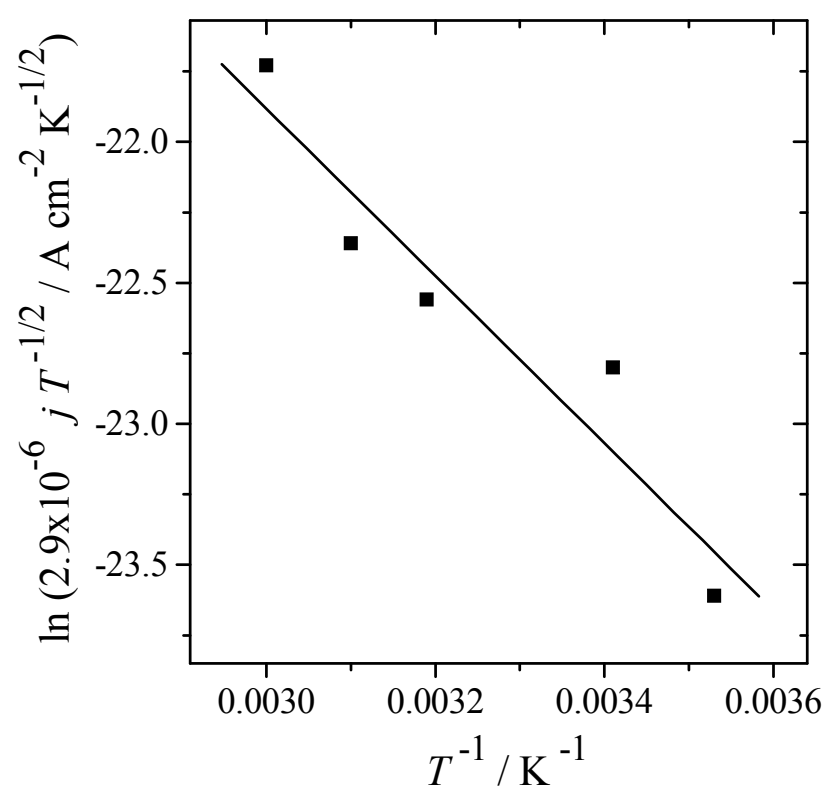

a)

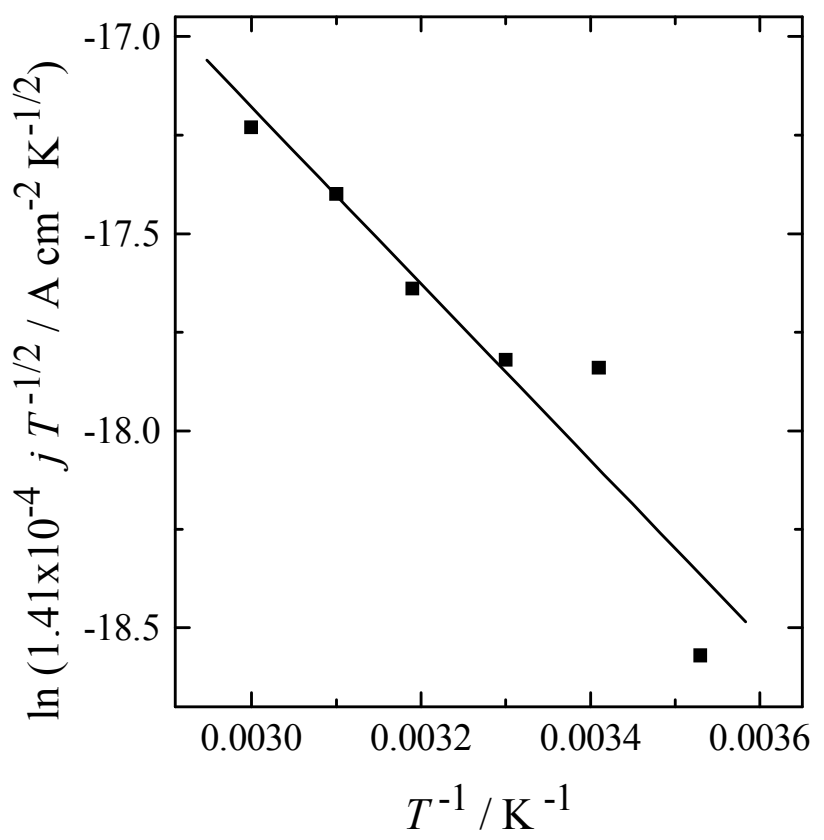

b)

Figura 5. Determinación gráfica de las entropías y entalpías de activación: a) para la electropolimerización en carbonato de propileno; b) para la electropolimerización en acetonitrilo. 
Las Fig. 5a y 5b son las representaciones gráficas de las Eq. 7 y 8, respectivamente. La regresión lineal conduce a los valores: $-12.68=\Delta \mathrm{S}_{\text {elp }}^{ \pm} / \mathrm{R}, \mathrm{y}$ $3054=\left(\Delta \mathrm{H}^{ \pm}{ }_{\text {elp }}-0.25 \mathrm{FE}\right) / \mathrm{R}$, para la polimerización en $\mathrm{CP}, \mathrm{y},-10.46=\Delta \mathrm{S}_{\text {elp }}^{ \pm} / \mathrm{R}, \mathrm{y}$ $2239=\left(\Delta \mathrm{H}_{\text {elp }}^{ \pm}-0.75 \mathrm{FE}\right) / \mathrm{R}$, para la polimerización en $\mathrm{AN}$. Tomando como potencial de referencia para medir E el valor del potencial al pie de pico (es decir, el potencial de referencia es $1.7 \mathrm{~V}_{\mathrm{AgQRE}}$ en $\mathrm{AN}$ (ver Fig. 4a) y $\left.1.25 \mathrm{~V}_{\mathrm{Ag}}\right|_{\mathrm{AgCl}}$ en $\mathrm{CP}$ (ver Fig. 4b)), se tiene $\mathrm{E}=0.34 \mathrm{~V}$ en $\mathrm{CP}, \mathrm{y} \mathrm{E}=0.45 \mathrm{~V}$ en $\mathrm{AN}$. Con estos datos se calculan los valores siguientes para $\Delta \mathrm{H}^{ \pm}$elp y $\Delta \mathrm{S}^{ \pm}$elp en CP y AN: 33.6 $\mathrm{kJ} / \mathrm{mol}$ y $-105 \mathrm{~J} / \mathrm{molK}$, en CP, y $25.3 \mathrm{~kJ} / \mathrm{mol}$ y $-87 \mathrm{~J} / \mathrm{molK}$ en $\mathrm{AN}$.

Como puede verse, la entalpía de activación en CP es un 25\% más elevada que la que se estima en AN. Por otra parte, las entropías de activación en ambos disolventes son negativas, indicando que, en ambos casos, el complejo activado está más ordenado que el monómero solvatado del que se parte, lo que sería de esperar en un proceso de poliadición radicálica a partir del cual se postula la formación del polímero.

\section{Mecanismos de polimerización}

Las características generales de los voltamogramas registrados en $\mathrm{CP}$ y $\mathrm{AN}$ no difieren de forma significativa entre sí: en ambos casos los voltamogramas no muestran picos de reducción durante el barrido hacia potenciales más negativos, lo que indica que el polímero se forma durante el barrido de oxidación, desprendiéndose, como se observa directamente, de la superficie del electrodo. No obstante, existen diferencias significativas en los valores de los parámetros cinéticos experimentales que indican que los mecanismos de formación se producen a través de trayectorias de reacción diversas. Los parámetros más divergentes entre sí son los valores de n $\alpha, 0.25$ en $\mathrm{CP}$ y 0.75 en $\mathrm{AN}, \mathrm{y}$, más

significativamente, los valores del orden de reacción con respecto a la concentración de monómero $\mathrm{Z}_{[\mathrm{FA}}$, que en $\mathrm{CP}$ vale 0.5 , y en $\mathrm{AN} 1$. Un orden de reacción fraccionario es característico de reacciones electroquímicas que tienen lugar en la capa adsorbida, mientras que un valor entero unido a uno de n $\alpha=$ 
0.75, que puede ser, simplemente, un factor de simetría $\beta$, parece indicar que la etapa determinante es una primera etapa de oxidación monoelectrónica del monómero desde la disolución (OHP) para dar un radical que actúa como iniciador de una polimerización que se produce en disolución, mientras que en CP la polimerización tiene lugar en la capa adsorbida. Ambos mecanismos se pueden formular del siguiente modo:

\section{En CP:}

Etapa 1. Adsorción de las moléculas de monómero sobre la superficie del electrodo:

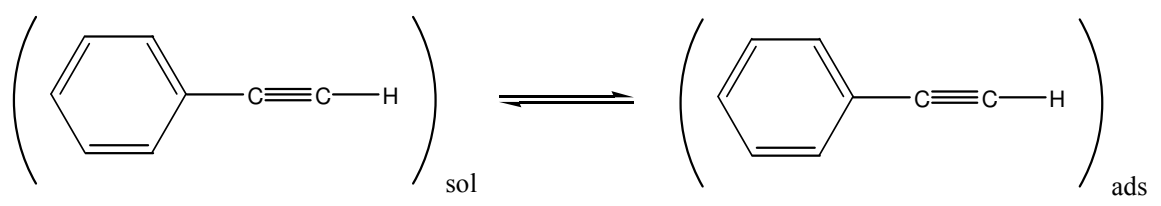

Etapa 2. Transferencia de carga desde la capa adsorbida (IHP) que provoca la ruptura del enlace $\equiv \mathrm{C}-\mathrm{H}$, para dar lugar a la formación del radical que actúa como iniciador de la polimerización. Esta es la etapa que se considera como determinante de la velocidad (rds). Considerar a esta etapa como determinante de la velocidad conduce a obtener una ecuación de velocidad teórica que coincide con la experimental. Además es la única etapa del mecanismo en la que se produce una ruptura de enlace (el enlace $\equiv \mathrm{C}-\mathrm{H}$ ), lo que significa que la energía de activación es más elevada que la de cualquiera de las etapas siguientes que son etapas de adición radicálica caracterizadas por meras reorganizaciones electrónicas. Por lo tanto:
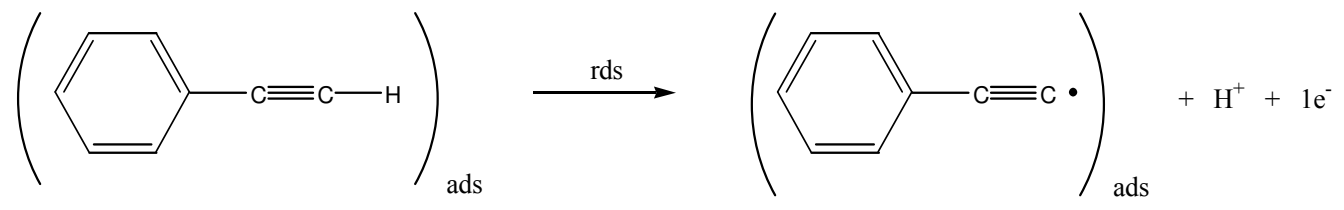

Etapa 3. Adición de un radical a una molécula de fenilacetileno en disolución para formar un radical dímero: 


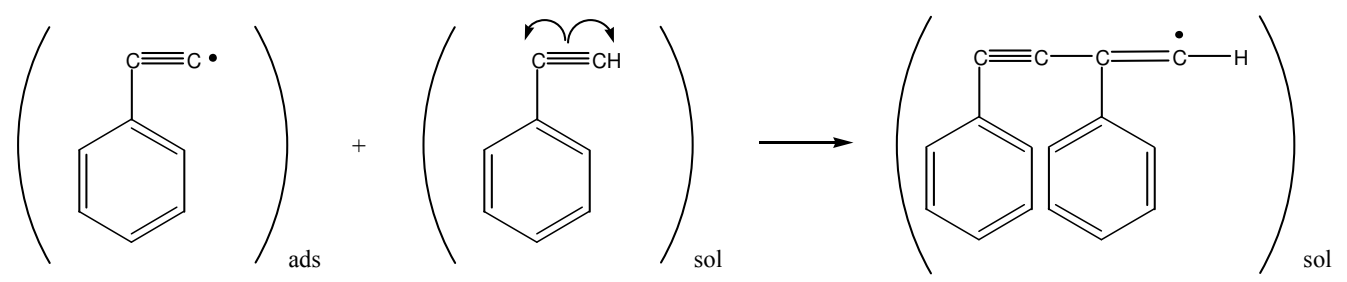

Etapa 4. Poliadición de moléculas de fenilacetileno:
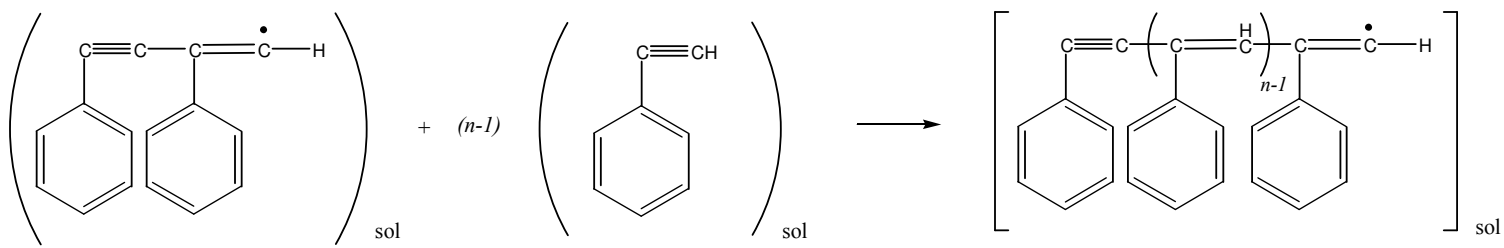

Etapa 5. Terminación:
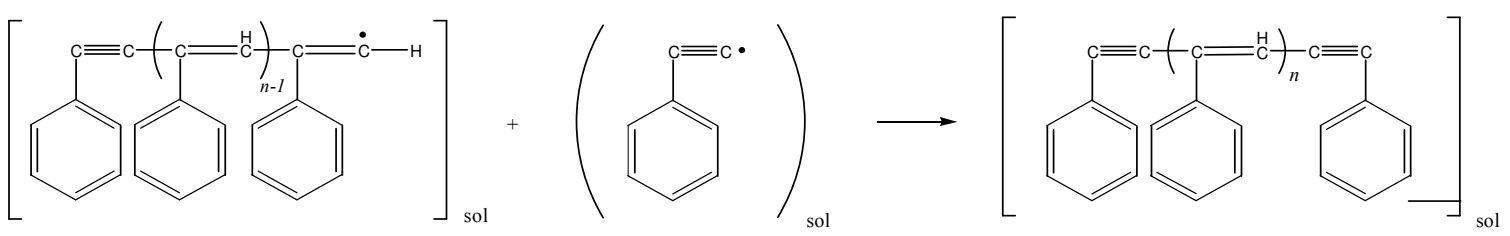

En el caso de la polimerización en $\mathrm{AN}$, el valor $\mathrm{Z}_{\mathrm{FA}}=1$ solo se puede explicar si la primera etapa del mecanismo es la reacción de formación del radical feniletinilo que actúa como iniciador de una polimerización por adición radicálica, y si dicha reacción, que ha de ser una transferencia de carga monoelectrónica con ruptura de enlace se produce desde el OHP, lo que excluye la adsorción previa del FA sobre el electrodo. Además, esta etapa es la determinante de la velocidad (edv), ya que la ruptura de un enlace $\equiv \mathrm{C}-\mathrm{H}$ va asociada a una barrera de energía de activación elevada. La ausencia de adsorción de las moléculas de FA se explica por que la fuerte interacción de adsorción de las moléculas de AN sobre los puntos activos superficiales ejerce una muy efectiva acción de bloqueo de estos con respecto a la adsorción de moléculas de monómero, por lo que la oxidación conducente a la formación del 
iniciador solo puede tener lugar desde la disolución. Por lo tanto, el mecanismo que se propone para la electropolimerización en AN es el siguiente:

Etapa 1. Las moléculas del monómero FA situadas en el OHP transfieren un electrón al electrodo dando lugar a la formación del iniciador en el OHP. En este caso esta primera etapa es la que se considera como determinante de la velocidad, con lo que ésta resulta proporcional a la concentración de FA, y a exp $\left(\frac{n \alpha F E}{R T}\right)$, con $n \alpha=\beta=0.75$. De este modo se obtiene directamente la ecuación de velocidad teórica, que coincide con la Eq. (4):

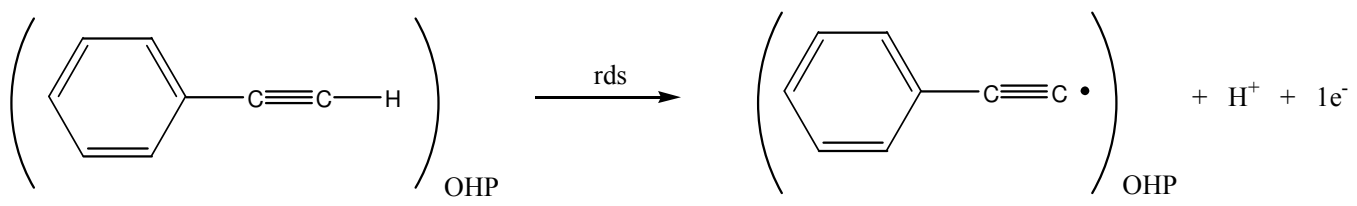

Etapa 2. Iniciación de la polimerización por adición con formación del dímero:

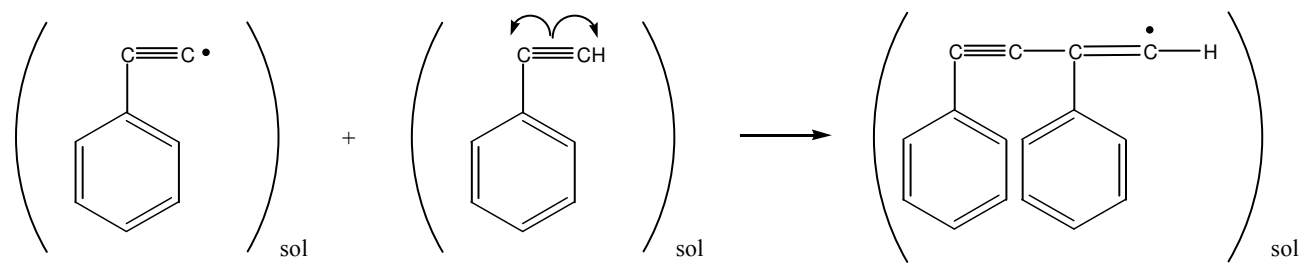

Etapa 3. Poliadición :
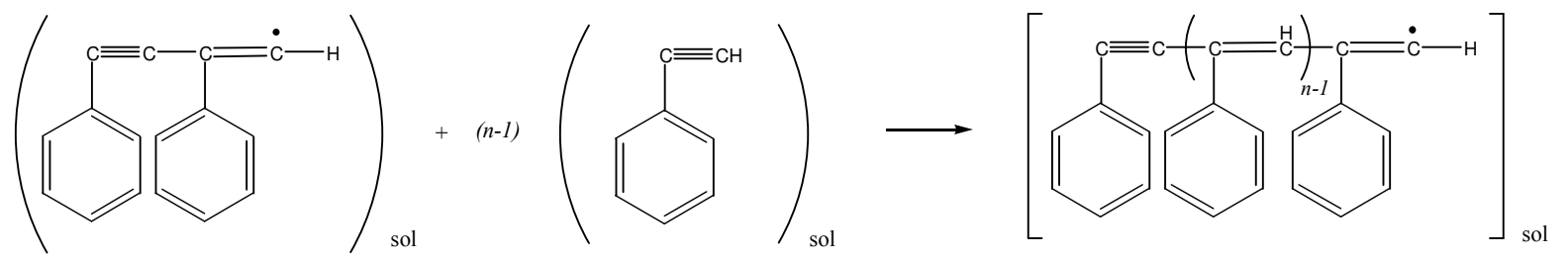

Etapa 4. Finalización: 

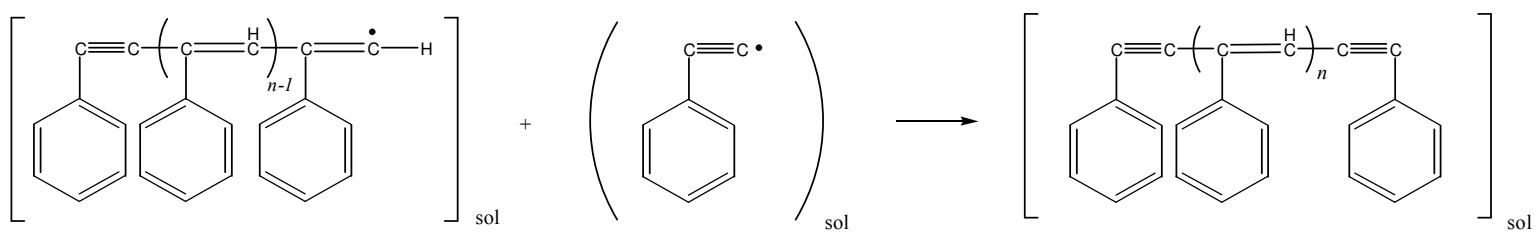

El tratamiento teórico del mecanismo que tiene lugar en $\mathrm{CP}$ se hace partiendo de considerar la ecuación [14]:

$$
\mathrm{v}_{\mathrm{p}}=\mathrm{k}_{\mathrm{p}}\left[\frac{\mathrm{v}_{\mathrm{i}}}{\mathrm{k}_{\mathrm{t}}}\right]^{1 / 2} \theta_{\mathrm{FA}}
$$

donde $\mathrm{k}_{\mathrm{p}}$ es la constante de velocidad de polimerización, $\mathrm{v}_{\mathrm{i}}$ la velocidad de la etapa de iniciación, que es la etapa $2, \mathrm{k}_{\mathrm{t}}$ la constante de velocidad de la etapa de terminación y $\theta_{\mathrm{FA}}$ el recubrimiento superficial con moléculas de monómero.

Aplicando la isoterma de Temkin para escribir las ecuaciones cinéticas correspondientes a las dos primeras etapas del mecanismo propuesto, se llega a la siguiente ecuación para $\mathrm{v}_{\mathrm{p}}$ :

$$
\mathrm{v}_{\mathrm{p}}=\frac{\mathrm{j}}{\mathrm{nF}}=\mathrm{K} \exp \left[\frac{\mathrm{g} \theta_{\mathrm{T}}}{2 \mathrm{RT}}\right] \exp \left[\frac{(1-\beta) \mathrm{FE}}{\mathrm{RT}}\right]
$$

Asimismo siguiendo el tratamiento cinético de la isoterma de Temkin:

$$
\exp \left[\frac{\mathrm{g} \theta_{\mathrm{T}}}{\mathrm{RT}}\right]^{1 / 2}=\mathrm{K}_{1}{ }^{1 / 2}[\mathrm{FA}]^{1 / 2}
$$

la cual, substituida en la Eq. (10) lleva a la ecuación:

$$
\mathrm{j}=\mathrm{nFKK}_{1}^{1 / 2}[\mathrm{FA}]^{1 / 2} \exp \left[\frac{(1-\beta) \mathrm{FE}}{\mathrm{RT}}\right]
$$

que coincide con la ecuación de velocidad experimental, Eq. (3).

\section{Conclusiones}

Los estudios electrocinéticos de la polimerización de fenilacetileno en carbonato de propileno y en acetonitrilo sobre electrodos de Pt conducen a valores de los parámetros cinéticos diferentes en ambos medios. Dichas diferencias pueden ser 
justificadas teóricamente proponiendo trayectorias de reacción que desembocan, en ambos medios, en una polimerización por poliadición radicálica. Las diferencias provienen de que la etapa de formación del iniciador de la polimerización tiene lugar en la capa adsorbida cuando se trabaja en carbonato de propileno, o en la disolución cuando se utiliza acetonitrilo como disolvente. Ello es debido a que, al contrario que el carbonato de propileno, las moléculas de acetonitrilo se adsorben fuertemente sobre la superficie electródica, impidiendo la adsorción de moléculas de fenilacetileno.

El polímero obtenido se caracterizó espectroscópicamente (IR, ${ }^{1} \mathrm{H}$ NMR, ${ }^{13} \mathrm{C}$ RMN. UV-VIS), lo que permitió deducir que estaba formado por cadenas poliénicas lineales con estructura trans.

\section{Agradecimientos}

Los autores desean expresar su agradecimiento al Ministerio de Ciencia y Tecnología de España por la concesión del Proyecto BQU 2002-02522, que hizo posible la realización de este trabajo.

Estudio de la Electropolimerización de Fenilacetileno sobre Electrodo de Pt en Disoluciones de Carbonato de Propileno y de Acetonitrilo

\section{Referencias}

1. Y. Kishimoto, P. Eckerle, T. Miyatake, M. Kainosho, A. Ono, T. Ikariya, R. Noyori, J. Am. Chem. Soc. 121 (1991) 12035.

2. C.I. Simionescu, V. Percec, Prog. Polym. Sci. 8 (1982) 133.

3. C.I. Simionescu, M. Grovu, Angew. Makromol. Chem. 111 (1983) 149.

4. R.W. Subramanian, J. Jakubowski, B.K. Garg, Polym. Prep. 19 (1978) 517.

5. C.I. Simionescu, M. Grovu, A. Duca, Angew. Makromol. Chem. 115 (1983) 47. 
6. J.C.W. Chien, "Polyacetylene Chemistry, Physics and Materials Science", Academic Press, Orlando FL (1984).

7. N. Sasaki, T. Masuda, T. Higashimura, Macromolecules 9 (1976) 664.

8. T. Masuda, N. Sasaki, T. Higashimura, Macromolecules 8 (1975) 717.

9. C.I. Simionescu, V. Percec, J. Polym. Sci. Polym. Symp. 67 (1980) 43.

10. V. Percec, P.L. Rinaldi, Polymer Bull. 9 (1983) 548.

11. A. Niki, T. Masuda, T. Higashimura, J. Pol. Sci. Part A, 25 (1987) 1553.

12. M.G. Evans, N.S. Hush, J. Chim. Phys. 49 (1952) 159.

13. J. González Velasco, J. Phys. Chem. B, en prensa.

14. K.J. Laidler, "Reaction Kinetics", Pergamon Press, Oxford (1966). 